\title{
Epithelial cyst of the iris following penetrating keratoplasty
}

\author{
S. ARTHUR BORUCHOFF, KENNETH R. KENYON, \\ GARY N. FOULKS, AND W. RICHARD GREEN \\ From the Cornea Service of the Massachusetts Eye and Ear Infirmary, Boston, Massachusetts, \\ and the Eye Pathology Laboratory of the Wilmer Ophthalmological Institute, Baltimore, Maryland, USA
}

SUMMARY In a 35-year-old patient with keratoconus a cyst of the iris was first observed 22 months after routine penetrating keratoplasty. The lesion was completely excised and shown by light and transmission electron microscopy to be composed of conjunctival epithelium organised as a single large cyst containing serous material and occasional free epithelial cells. The management of epithelial implantation cysts is discussed. When indicated, complete surgical excision without rupture is preferred to avoid possible intraocular dissemination of viable epithelial cells that might result in further complications.

\begin{abstract}
Aberrant epithelium in the ocular anterior chamber is a well recognised complication of accidental and surgical perforating wounds of the cornea. Epithelialisation involving the iris may develop as (1) solid (pearl) tumours, (2) serous cysts, (3) epithelial implantation membranes, or (4) epithelial ingrowth. ${ }^{12}$ Although each type of epithelial invasion shows different growth characteristics, all have a potential for progression, which in some cases can be rapid and extensive, resulting in visual obstruction, iridocyclitis, secondary glaucoma, and potential loss of the eye. ${ }^{12}$ The decision to intervene surgically is predicated on documented progression of the lesion and knowledge of its potential dangers. The preferred method of treatment must be based on the site affected and anticipation of the behaviour of the lesion.

We observed the occurrence and progression of a cystic lesion of the iris following penetrating keratoplasty. Treatment by excision provided the first histopathological specimen to be examined by transmission electron microscopy.
\end{abstract}

\section{Case report}

A 35-year-old Caucasian male required penetrating keratoplasty for keratoconus. His eye was otherwise normal. Penetrating keratoplasty was performed on the left eye without apparent complication, with continuous 10-0 nylon suture placed at midstromal

Correspondence to S. A. Boruchoff, MD, Massachusetts Eye and Ear Infirmary, 243 Charles Street, Boston, Massachusetts 02114, USA. depth. A routine follow-up 15 months after surgery showed a clear graft, visual acuity corrected to $20 / 20(6 / 6)$ with spectacles, and no abnormalities. The continuous nylon suture was removed uneventfully during the $22 \mathrm{nd}$ postoperative month, at which time a translucent cystic elevation of the iris about $2.5 \mathrm{~mm}$ in diameter was noted at the 3 o'clock position underlying the keratoplasty wound without corneal touch (Fig. 1). The pupil was slightly elliptical, but no extension of the lesion into the angle was apparent.

During a 3-month interval progression in size to about $4 \mathrm{~mm}$ was documented, and during the 25th month after keratoplasty the lesion was excised. A fornix-based conjunctival flap was dissected temporally. An incision was made at the limbus and extended with scissors from 2 to 4 o'clock. To avoid rupture of the cyst within the eye a Weck-cel sponge was applied to the cyst for traction, and the cyst was withdrawn from the anterior chamber. Radial section of the iris above and below the lesion was made from the angle to the pupillary margin, the base of the iris was transected, and the cyst was removed intact. The entire specimen was immediately fixed in $4 \%$ glutaraldehyde (cacodylate-buffered) and prepared for transmission electron microscopy by standard techniques.

Postoperatively the graft has remained clear with visual acuity correctable to $20 / 20(6 / 6)$. Corneal astigmatism of approximately 6 dioptres before excision was reduced to 2.75 dioptres after the operation. Although we were concerned that the large temporal iridectomy might produce glare 


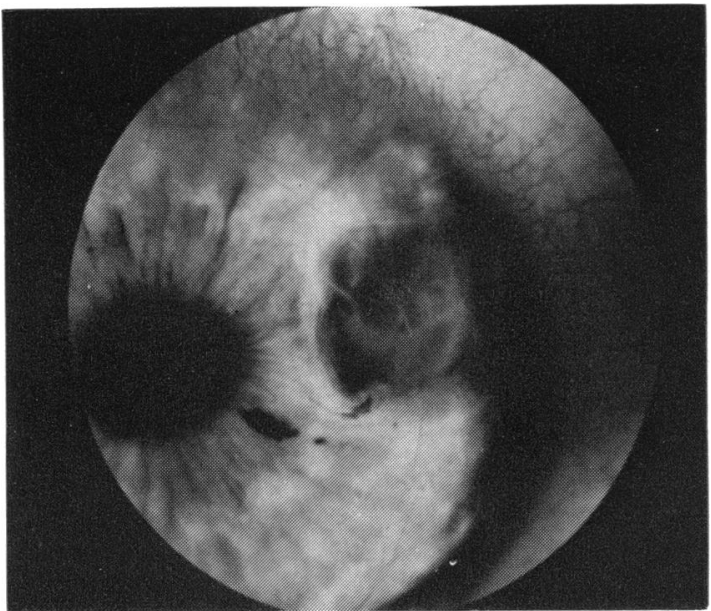

Fig. 1 Appearance of thin-walled iris cyst 22 months after penetrating keratoplasty. Surgical wound margin between donor and recipient corneas is apparent.

symptoms, the patient has not experienced such problems. There has been no sign of inflammation, glaucoma, or recurrent anterior chamber epithelialisation during the past 36 months.

\section{PATHOLOGICAL FINDINGS}

Gross examination. The excised iris specimen contained a thin-walled cystic structure, measuring 3.0 $\mathrm{mm}$ in diameter, which transilluminated easily.

Light microscopy. A solitary cyst lined by nonkeratinised stratified squamous epithelium of variable thickness was almost completely embedded within the iris stroma (Fig. 2). Nasally and temporally the cyst appeared to dissect between the layers of stroma. Only a very thin layer of stroma was present between the cyst and the intact but attenuated dilator muscle and pigment epithelium of the iris. The epithelial lining was thicker nasally, temporally, and posteriorly, where it attained up to 10 cell layers (Fig. 3 inset). The epithelium was thinnest anteriorly, as was the extremely diaphenous iris stroma covering it. Nasally a nodule of the acanthotic epithelium showed desquamation of its surface cells (Fig. 4 inset). The contents of the cyst appeared almost entirely serous, although an occasional rounded-up, desquamated epithelial cell was also apparent.

Electron microscopy. Survey of the posterior aspect of the cyst revealed 4 to 5 layers of stratified epithelial cells with occasional melanocytes interspersed among the basal cell layer (Fig. 3). The iris stroma, dilator muscle, and pigment epithelium appeared ultrastructurally unremarkable. The basal epithelial cells possessed a thin, discontinuous basement membrane with relatively few hemidesmosomal attachments. Cells of all epithelial layers contained numerous tonofilaments and several small melanosomes. The superficial cells showed innumerable microvillous projections of their free surface plasma membrane (Fig. 3 inset). Fine filamentous material, similar to the glycoprotein normally adherent to ocular surface epithelial cells, invested the surface of the microvilli. On the basis of these several ultrastructural criteria this epithelium could be judged to have originated from the limbus or bulbar conjunctiva.

Of particular interest was the nodular area of desquamating epithelial cells. Several apparently free-floating cells could be examined, and all appeared entirely viable, as they had intact plasma membranes and cytoplasmic organelles with no evidence of senescent or degenerative changes (Fig. 4). In this same area, several intraepithelial pseudocysts were also apparent (Fig. 5). The cells lining these pseudocysts had retained the corrugated characteristics of their surface microprojections, and the material within the cystic cavities was finely reticulated and similar to that adherent to the plasma membrane surface (Fig. 5 inset). Multiple small spherules (about $0.1 \mathrm{um}$ diameter) were also among the contents of the pseudocysts and resembled the secretory vesicles frequently evident in corneal and conjunctival surface cells. A similar electron microscopic configuration has been observed in other intraepithelial pseudocysts of the conjunctiva. $^{3}$

\section{Discussion}

The incidence of epithelialisation of the anterior chamber after penetrating ocular injury or surgery

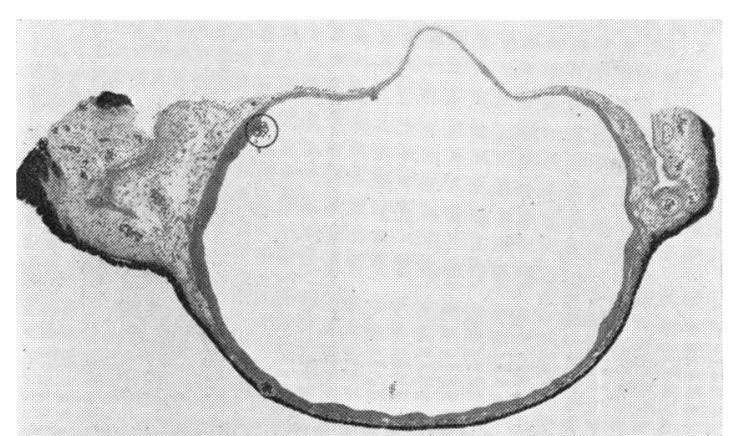

Fig. 2 Light micrograph of entire surgical specimen discloses single cyst with epithelial lining and serous contents implanted in anterior iris stroma. Nasally a nodule of the lining epithelium shows desquamation of its surface cells (circled)(paraphenylenediamine; $\times 16)$. 


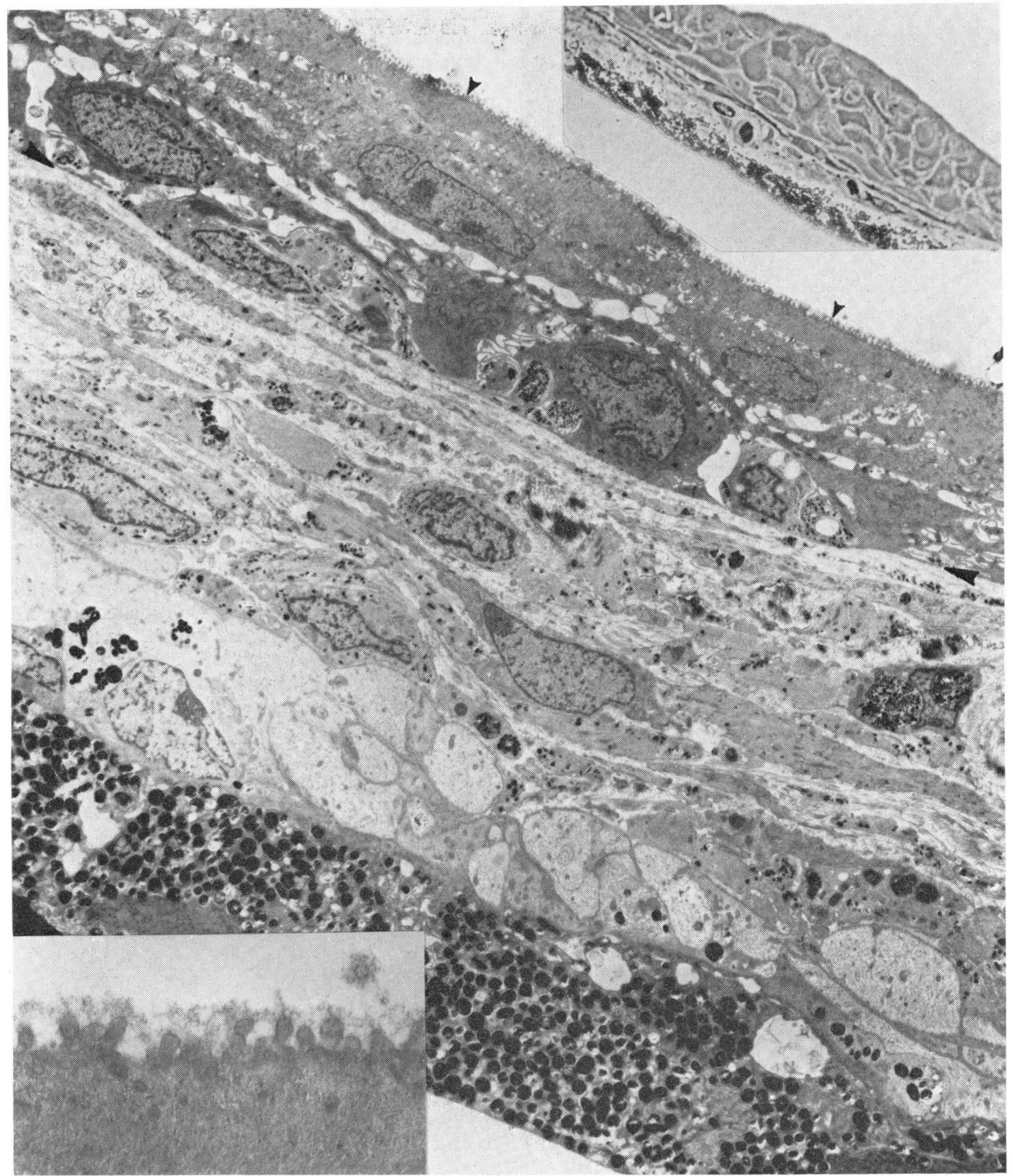

Fig. 3 Inset (top right) is light micrograph of posterior wall of lesion, showing multiple layers of stratified, nonkeratinising epithelium overlying attenuated iris stroma, dilator muscle, and pigment epithelium (paraphenylenediamine, phase contrast; $\times 400$ ). Main figure electron micrograph of same area illustrates numerous surface microprojections (small arrowheads) of epithelial cells exposed to lumen of cyst, small nielanosomes within intermediate and basal cells, and melanocytes along basement membrane plane (large arrowheads). Normal appearing iris stroma, dilator muscle, and pigment epithelial cell layers are included $(\times 3000)$. Inset (bottom left $)$ is higher magnification of epithelial cell surface to resolve microvilli with adherent filamentous material $(\times 25000)$. 
has been recorded as $0.06 \%{ }^{4}$ in one large series of 45500 patients. In a clinical series of 8.062 cataract extractions Theobald and $\mathrm{Haas}^{5}$ reported an incidence of $0.11 \%$ of anterior chamber epithelial invasion. Reports of epithelialisation of the anterior chamber following keratoplasty are rare. Leibowitz et al. ${ }^{6}$ demonstrated the presence of retrocorneal epithelial cells in a patient with delayed wound healing and iris adhesion to the wound who subsequently developed an epithelial cyst. Bennett and

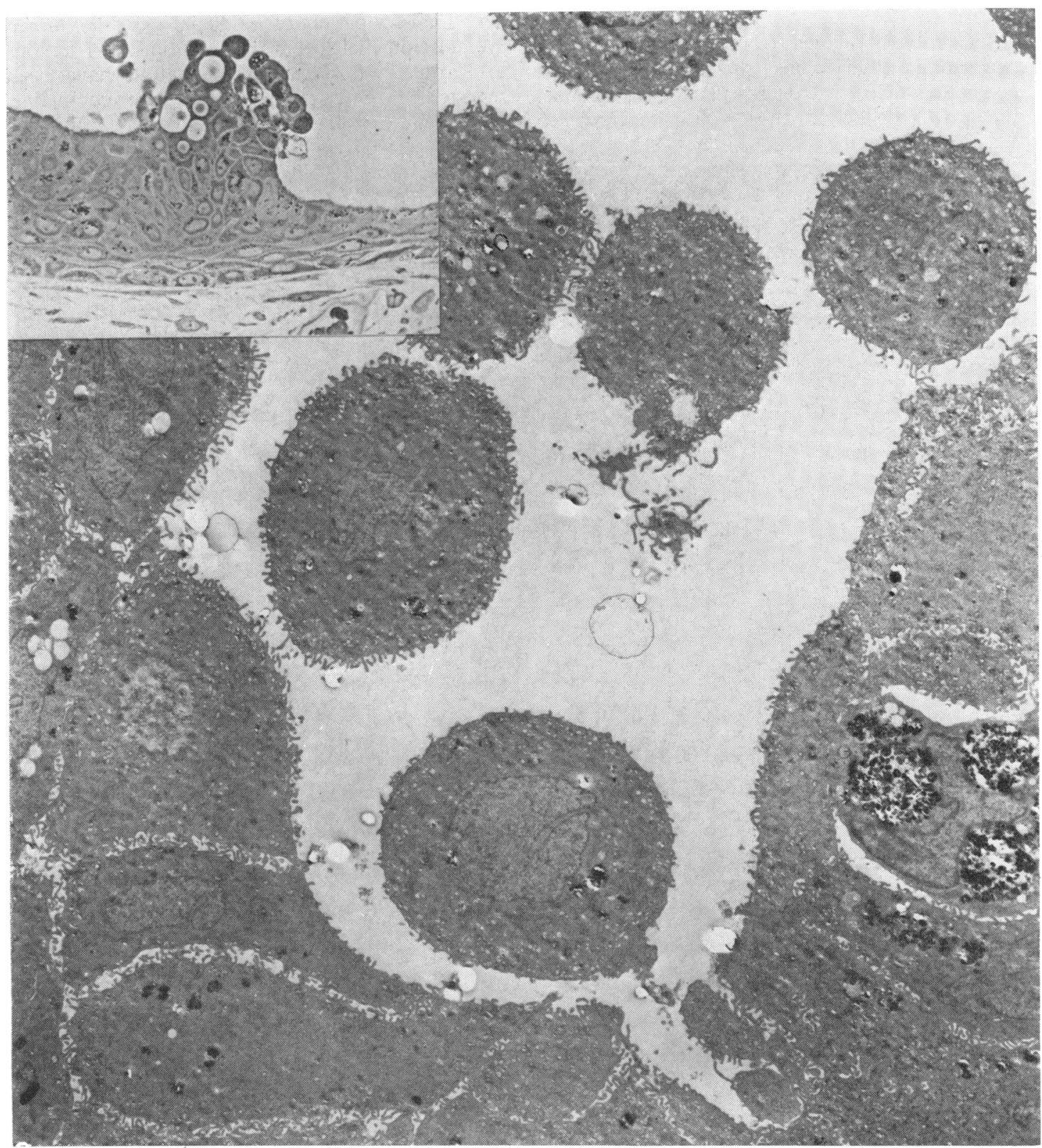

Fig. 4 Inset light micrograph illustrates nodule of acanthotic epithelium exhibiting surface desquamation and intraepithelial cyst formation (paraphenylenediamine, phase contrast; $\times 400$ ). Main figure electron micrograph shows these desquamating surface cells to appear normal and viable with respect to intactness of plasma membranes and cytoplasmic contents $(\times 4000)$. 
D'Amico ${ }^{7}$ also reported an epithelial inclusion cyst that was verified histopathologically following keratoplasty in an eye with episodic iritis. Experimentally, epithelialisation has been produced as ingrowth, pearl tumour, and implantation membranes but not as an isolated implantation cyst. $^{89}$

It is generally agreed from both clinical and experimental observations that the onset and exacerbation of epithelial growth is unpredictable. While growth is likely to be slow, it is imperative to observe epithelial lesions in the anterior chamber regularly for signs of progression. If such progression is reported, then surgical intervention is indicated. The goal of therapy is complete excision of the lesion if possible without release of free, potentially viable epithelial cells into the anterior chamber. Transformation of epithelial cysts in human eyes to epithelial ingrowth and from pearl tumour to serous cyst after instrumentation have been reported..$^{11}$ Subjective postoperative complaints such as glare or distortion are secondary considera-

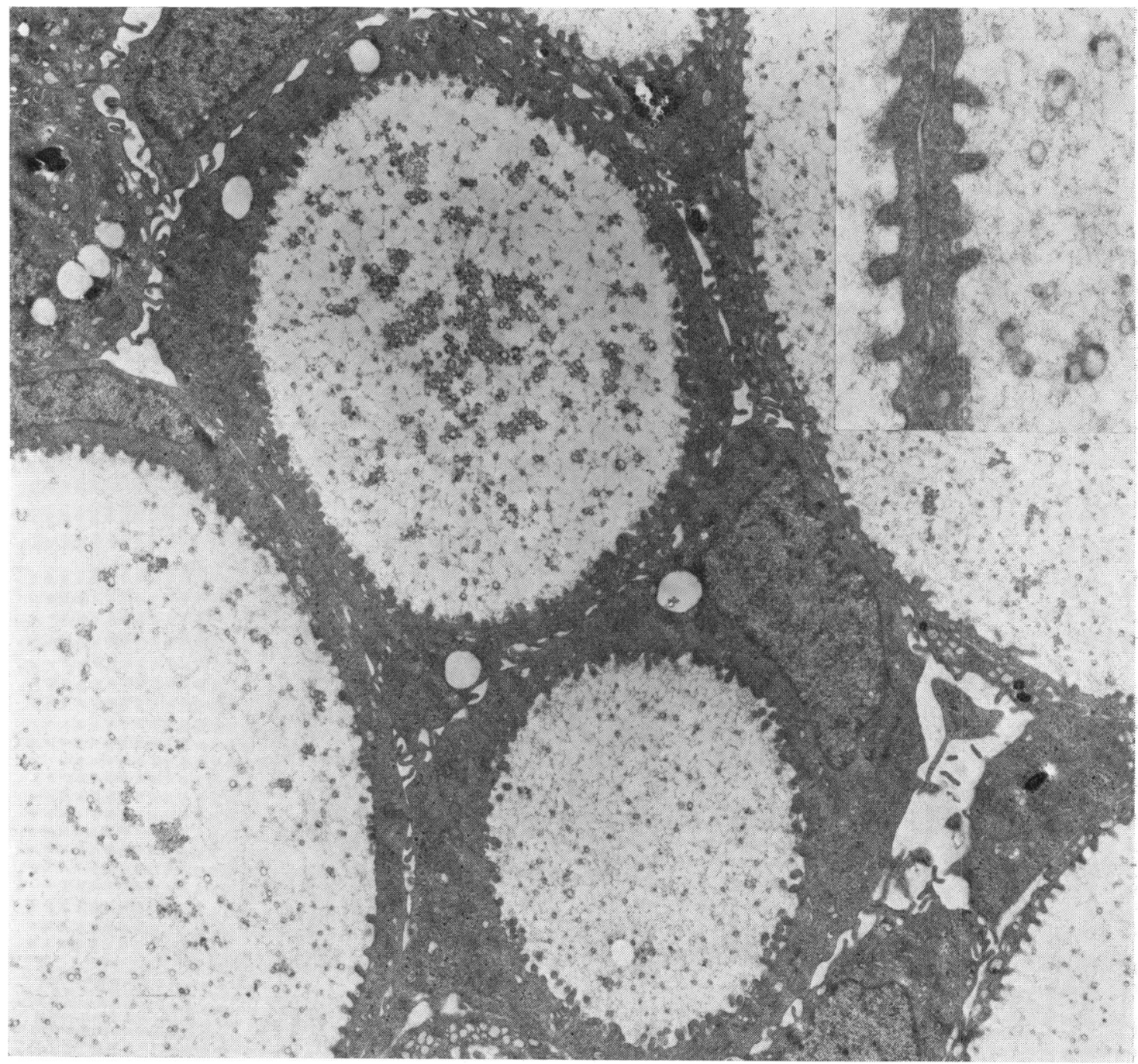

Fig. 5 Electron micrograph of cystic area reveals multiple intraepithelial pseudocysts, lined by nonkeratinised epithelium and containing fine granular and vesicular material $(\times 7000)$. Inset focuses on 2 attenuated epithelial cell processes demarcating adjacent pseudocysts. These cells exhibit microvillous surface projections of their plasma membranes that are coated with fine filaments appearing similar to the contents of the cysts. Vesicular material is presumably glycoprotein or mucopolysaccharide secreted by these epithelial cells $(\times 33000)$. 
tions in view of the devastation that intraocular epithelial proliferation might cause.

In the case reported here a serous cyst of the iris followed an apparently uncomplicated penetrating keratoplasty without the predisposing features of wound healing problems, iris adhesion, hypotony, or epithelial communication with the wound. We believe the case represents an implantation of conjunctival epithelium rather than ingrowth. We concur with Ferry ${ }^{12}$ and Bennett and D'Amico ${ }^{7}$ concerning the possibility of inadvertent implantation of free epithelial cells by forceps or surgical instruments during keratoplasty.

Many therapeutic approaches have been advocated for managing epithelial cysts in the anterior chamber. Aspiration (with or without cauterisation), while sometimes successful, is usually only a temporising measure, since recurrence is common and can lead to epithelial downgrowth. ${ }^{10}$ Marsupialisation, photocoagulation, electrolysis, and cryothermy suffer similar disadvantages. Radical anterior segment reconstruction, while perhaps necessary for complete excision of an extensive lesion, may not be required if the lesion can be removed in toto while still small. ${ }^{13}$

The authors gratefully acknowledge the technical assistance of $\mathrm{Mr}$ Randy Kimsey in the preparation of the electron micrographs.

Supported in part by a Bausch and Lomb Cornea fellowship (Dr Kenyon) and a postdoctoral National Research Service award from the National Eye Institute (Dr Foulks).

\section{References}

${ }^{1}$ Maumenee AE, Shannon CR. Epithelial invasion of the anterior chamber. Am J Ophthalmol 1956; 41: 929-42.

${ }^{2}$ Friedman AH, Taterka HB, Henkind P. Epithelial implantation membrane on the iris surface following cataract extraction with report of two cases. Am J Ophthalmol 1971; 71 : $482-5$.

${ }^{3}$ Tripathi RC, Bron AJ. Cystic disorders of the corneal epithelium. II. Pathogenesis. Br J Ophthalmol 1973; 57: 376-90.

${ }^{4}$ Terry TL, Chisholm JF, Schonberg AL. Studies on surfaceepithelium invasion of the anterior segment of the eye. Am J Ophthalmol 1939; 22: 1083-108.

${ }^{5}$ Theobald GD, Haas JS. Epithelial invasion of the anterior chamber following cataract extraction. Trans Am Acad Ophthalmol Otolaryngol 1948; 53: 470-82.

'Leibowitz HM, Elliot JH, Boruchoff SA. Epithelialization of the anterior chamber following penetrating keratoplasty. Arch Ophthalmol 1967; 78: 613-7.

'Bennett T, D'Amico RA. Epithelial inclusion cyst of iris after keratoplasty. Am J Ophthalmol 1974; 77: 87-9.

${ }^{8}$ Patz A, Wulff L, Rogers S. Experimental production of epithelial invasion of the anterior chamber. Am J Ophthalmol 1959; 47: 815-27.

${ }^{9}$ Regan E. Epithelial invasion of the anterior chamber. Arch Ophthalmol 1958; 60: 907-27.

${ }^{10} \mathrm{Harbin}$ TS, Maumenee AE. Epithelial downgrowth after surgery for epithelial cyst. Am J Ophthalmol 1974; 78: 1-4.

${ }^{11}$ Kinoshita A. A case of traumatic iris cyst. Folia Ophthalmol Jpn 1967; 18: 776-9.

${ }^{12}$ Ferry AP. The possible role of epithelium-bearing surgical instruments in pathogenesis of epithelialization of the anterior chamber. Ann Ophthalmol 1971; 3: 1089-93.

${ }^{13}$ Friedman AH. Radical anterior segment surgery for epithelial invasion of the anterior chamber: report of three cases. Trans Am Acad Ophthalmol Otolaryngol 1977; 83: 216-23. 